\title{
ON THE SPACES OF FIBONACCI DIFFERENCE ABSOLUTELY $p$-SUMMABLE, NULL AND CONVERGENT SEQUENCES
}

\author{
METIN BAŞARIR, FEYZİ BAŞAR AND EMRAH EVREN KARA
}

\begin{abstract}
Let $0<p<1$. In the present paper, as the domain of the band matrix $\widehat{F}$ defined by the Fibonacci sequence in the classical sequence spaces $\ell_{p}, c_{0}$ and $c$, we introduce the sequence spaces $\ell_{p}(\widehat{F})$, $c_{0}(\widehat{F})$ and $c(\widehat{F})$, respectively. Also, we give some inclusion relations and construct the bases of the spaces $c_{0}(\widehat{F})$ and $c(\widehat{F})$. Finally, we compute the alpha, beta, gamma duals of these spaces and characterize the classes $\left(\ell_{p}(\widehat{F}), \mu\right)$ of infinite matrices with $\mu \in\left\{\ell_{\infty}, c, c_{0}\right\}$.
\end{abstract}

\section{INTRODUCTION}

By $\mathbb{N}$ and $\mathbb{R}$, we denote the sets of all natural and real numbers, respectively. Let $\omega$ be the vector space of all real sequences. Any vector subspace of $\omega$ is called a sequence space. Let $\ell_{\infty}, c, c_{0}$ and $\ell_{p}$ denote the classes of all bounded, convergent, null and absolutely $p$-summable sequences, respectively; where $0<p<\infty$. Moreover, we write $b s$ and $c s$ for the spaces of all bounded and convergent series, respectively. Also, we use the conventions that $e=(1,1,1, \ldots)$ and $e^{(n)}$ is the sequence whose only non-zero term is 1 in the $n^{\text {th }}$ place for each $n \in \mathbb{N}$.

Let $\lambda$ and $\mu$ be two sequence spaces, and $A=\left(a_{n k}\right)$ be an infinite matrix of real numbers $a_{n k}$, where $n, k \in \mathbb{N}$. Then, we say that $A$ defines a matrix transformation from $\lambda$ into $\mu$ and we denote it by writing $A: \lambda \rightarrow \mu$, if for every sequence $x=\left(x_{k}\right) \in \lambda$ the $A$-transform $A x=\left\{A_{n}(x)\right\}$ of $x$ is in $\mu$, where

$$
A_{n}(x)=\sum_{k} a_{n k} x_{k} \text { for each } n \in \mathbb{N} \text {. }
$$

2010 Mathematics Subject Classification. 11B39, 46A45, 46B45.

Key words and phrases. Fibonacci numbers, sequence spaces, difference matrix, alpha, beta and gamma duals, matrix transformations.

Copyright (c) 2016 by ANUBIH. 
For simplicity in notation, here and in what follows, the summation without limits runs from 0 to $\infty$. By $(\lambda, \mu)$, we denote the class of all matrices $A$ such that $A: \lambda \rightarrow \mu$. Thus, $A \in(\lambda, \mu)$ if and only if the series on the right side of (1.1) converges for each $n \in \mathbb{N}$ and every $x \in \lambda$, and we have $A x \in \mu$ for all $x \in \lambda$. Also, we write $A_{n}=\left(a_{n k}\right)_{k \in \mathbb{N}}$ for the sequence in the $n$-th row of $A$.

The domain $\lambda_{A}$ of an infinite matrix $A$ in a sequence space $\lambda$ is defined by

$$
\lambda_{A}:=\left\{x=\left(x_{k}\right) \in \omega: A x \in \lambda\right\}
$$

which is a sequence space. Depending on the choice of the matrix $A, \lambda_{A}$ may include the original space $\lambda$ or $\lambda_{A}$ may be included by the original space $\lambda$ and sometimes $\lambda_{A}$ may be identical to the space $\lambda$. Indeed if we choose $A=\Delta$, the backward difference matrix, then $c_{\Delta} \supset c$ but in the case $A=$ $\Delta^{-1}=S$, the summation matrix, $c_{S}=c s \supset c$, where both of two inclusions are proper. However, if we define $\lambda=c_{0} \oplus \operatorname{span}\{z\}$ with $z=\left\{(-1)^{k}\right\}$, i.e., $x \in \lambda$ if and only if $x=s+\alpha z$ for some $s \in c_{0}$ and some $\alpha \in \mathbb{C}$, and consider the matrix $A$ with the rows $A_{n}$ defined by $A_{n}=(-1)^{n} e^{(n)}$ for all $n \in \mathbb{N}$, we have $A e=z \in \lambda$ but $A z=e \notin \lambda$ which gives that $z \in \lambda \backslash \lambda_{A}$ and $e \in \lambda_{A} \backslash \lambda$. That is to say that the sequence spaces $\lambda_{A}$ and $\lambda$ overlap but neither contains the other.

By using the domain of a triangle infinite matrix, many sequence spaces have recently been defined by several authors, see for instance $[1,28,2,7]$. In the literature, the matrix domain $\lambda_{\Delta}$ is called the difference sequence space whenever $\lambda$ is a normed or paranormed sequence space, where $\Delta=\left(d_{n k}\right)$ denotes the backward difference matrix defined by

$$
d_{n k}=\left\{\begin{array}{cl}
(-1)^{n-k} & , \quad n-1 \leq k \leq n, \\
0 & , \quad 0 \leq k<n-1 \text { or } k>n
\end{array}\right.
$$

for all $k, n \in \mathbb{N}$. The notion of difference sequence spaces was introduced by Kizmaz [23], who defined the sequence spaces

$$
X(\Delta)=\left\{x=\left(x_{k}\right) \in \omega:\left(x_{k}-x_{k+1}\right) \in X\right\}
$$

for $X=\ell_{\infty}, c$ and $c_{0}$. The difference space $b v_{p}$, consisting of all sequences $\left(x_{k}\right)$ such that $\left(x_{k}-x_{k-1}\right)$ is in the sequence space $\ell_{p}$, was studied in the case $0<p<1$ by Altay and Başar [4] and in the case $1 \leq p \leq \infty$ by Başar and Altay [8], and Çolak et al. [12]. Kirişçi and Başar [24] have introduced and studied the generalized difference sequence spaces

$$
\widehat{X}=\left\{x=\left(x_{k}\right) \in \omega: B(r, s) x \in X\right\},
$$

where $X$ denotes any of the spaces $\ell_{\infty}, c, c_{0}$ and $\ell_{p}$, and $B(r, s) x=\left(s x_{k-1}+\right.$ $\left.r x_{k}\right)$ with $r, s \in \mathbb{R} \backslash\{0\}$, and $1 \leq p<\infty$. Following Kirişçi and Başar [24], Sönmez [33] have examined the sequence space $X(B)$ as the set of all 
sequences whose $B(r, s, t)$-transforms $B(r, s, t) x=\left(r x_{k}+s x_{k+1}+t x_{k+2}\right)$ are in the space $X \in\left\{\ell_{\infty}, \ell_{p}, c, c_{0}\right\}$ where $r, s, t \in \mathbb{R} \backslash\{0\}$. Also in $[14,32,29,18$, 9, 34, 17], authors studied certain difference sequence spaces. Furthermore, quite recently, Kara [21] has defined the Fibonacci difference matrix $\widehat{F}$ by means of the Fibonacci sequence $\left(f_{n}\right)_{n \in \mathbb{N}}$ and introduced the new sequence spaces $\ell_{p}(\widehat{F})$ and $\ell_{\infty}(\widehat{F})$ which are derived by the matrix domain of $\widehat{F}$ in the sequence spaces $\ell_{p}$ and $\ell_{\infty}$, respectively; where $1 \leq p<\infty$.

In this paper, we introduce the sequence spaces $\ell_{p}(\widehat{F}), c_{0}(\widehat{F})$ and $c(\widehat{F})$ by using the Fibonacci difference matrix $\widehat{F}$, where $0<p<1$. The rest of this paper is organized, as follows:

In Section 2, we give some notations and basic concepts. In Section 3, we introduce the sequence spaces $\ell_{p}(\widehat{F}), c_{0}(\widehat{F})$ and $c(\widehat{F})$, and establish some inclusion relations. Also, we construct the bases of these spaces. In Section 4 , the alpha, beta and gamma duals of the spaces $\ell_{p}(\widehat{F}), c_{0}(\widehat{F})$ and $c(\widehat{F})$ are determined, and the classes $\left(\ell_{p}(\widehat{F}), \ell_{\infty}\right),\left(\ell_{p}(\widehat{F}), c\right)$ and $\left(\ell_{p}(\widehat{F}), c_{0}\right)$ of matrix transformations are characterized.

\section{Preliminaries}

A sequence space $\lambda$ is called an $F K$-space if it is a complete linear metric space with continuous coordinates $p_{n}: \lambda \rightarrow \mathbb{C}$ with $p_{n}(x)=x_{n}$ for all $x=\left(x_{n}\right) \in \lambda$ and every $n \in \mathbb{N}$, where $\mathbb{C}$ denotes the complex field. A normed $F K$-space is called a $B K$-space, that is, a $B K$-space is a Banach space with continuous coordinates. For example, the space $\ell_{p}$ is a $B K$ space with $\|x\|_{p}=\left(\sum_{k}\left|x_{k}\right|^{p}\right)^{1 / p}$ and $c_{0}, c$ and $\ell_{\infty}$ are $B K$-spaces with $\|x\|_{\infty}=\sup _{k \in \mathbb{N}}\left|x_{k}\right|$, where $1 \leq p<\infty$. The sequence space $\lambda$ is said to be solid (cf. [20, p. 48]) if and only if

$$
\widetilde{\lambda}:=\left\{\left(u_{k}\right) \in \omega: \exists\left(x_{k}\right) \in \lambda \text { such that }\left|u_{k}\right| \leq\left|x_{k}\right| \text { for all } k \in \mathbb{N}\right\} \subset \lambda .
$$

A sequence $\left(b_{n}\right)$ in a normed space $X$ is called a Schauder basis for $X$ if for every $x \in X$ there is a unique sequence $\left(\alpha_{n}\right)$ of scalars such that $x=\sum_{n} \alpha_{n} b_{n}$, i.e., $\left\|x-\sum_{n=0}^{m} \alpha_{n} b_{n}\right\| \rightarrow 0$, as $m \rightarrow \infty$.

The alpha, beta and gamma duals $\lambda^{\alpha}, \lambda^{\beta}$ and $\lambda^{\gamma}$ of a sequence space $\lambda$ are respectively defined by

$$
\begin{aligned}
& \lambda^{\alpha}:=\left\{a=\left(a_{k}\right) \in \omega: a x=\left(a_{k} x_{k}\right) \in \ell_{1} \text { for all } x=\left(x_{k}\right) \in \lambda\right\}, \\
& \lambda^{\beta}:=\left\{a=\left(a_{k}\right) \in \omega: a x=\left(a_{k} x_{k}\right) \in c s \text { for all } x=\left(x_{k}\right) \in \lambda\right\}, \\
& \lambda^{\gamma}:=\left\{a=\left(a_{k}\right) \in \omega: a x=\left(a_{k} x_{k}\right) \in b s \text { for all } x=\left(x_{k}\right) \in \lambda\right\} .
\end{aligned}
$$


The sequence $\left(f_{n}\right)$ of Fibonacci numbers is defined by the linear recurrence equalities

$$
f_{0}=f_{1}=1 \text { and } f_{n}=f_{n-1}+f_{n-2} \text { with } n \geq 2 .
$$

Fibonacci numbers have many interesting properties and applications in arts, sciences and architecture. For example, the ratio sequences of Fibonacci numbers converge to the golden ratio which is important in sciences and arts. Some basic properties of sequences of Fibonacci numbers are also given, below (cf. Koshy [25]):

$$
\begin{aligned}
& \lim _{n \rightarrow \infty} \frac{f_{n+1}}{f_{n}}=\frac{1+\sqrt{5}}{2}=\varphi \text { (Golden Ratio), } \\
& \sum_{k=0}^{n} f_{k}=f_{n+2}-1 \text { for each } n \in \mathbb{N}, \\
& \sum_{k} \frac{1}{f_{k}} \text { converges, } \\
& f_{n-1} f_{n+1}-f_{n}^{2}=(-1)^{n+1} \text { for all } n \geq 1 \text { (Cassini Formula). }
\end{aligned}
$$

One can easily derive by substituting $f_{n+1}$ in Cassini's formula that $f_{n-1}^{2}+$ $f_{n} f_{n-1}-f_{n}^{2}=(-1)^{n+1}$.

Now, let $A=\left(a_{n k}\right)$ be an infinite matrix and $\mathcal{F}$ be the collection of all finite subsets of $\mathbb{N}$. We list the following conditions:

$$
\begin{aligned}
& \sup _{n \in \mathbb{N}} \sum_{k}\left|a_{n k}\right|<\infty \\
& \lim _{n \rightarrow \infty} a_{n k}=0 \text { for each } k \in \mathbb{N} \\
& \exists \alpha_{k} \in \mathbb{R} \ni \lim _{n \rightarrow \infty} a_{n k}=\alpha_{k} \text { for each } k \in \mathbb{N} \\
& \lim _{n \rightarrow \infty} \sum_{k} a_{n k}=0 \\
& \exists \alpha \in \mathbb{R} \ni \lim _{n \rightarrow \infty} \sum_{k} a_{n k}=\alpha \\
& \sup _{K \in \mathcal{F}} \sum_{n}\left|\sum_{k \in K} a_{n k}\right|<\infty \\
& \sup _{k, n \in \mathbb{N}}\left|a_{n k}\right|<\infty
\end{aligned}
$$

Now, we can give the following lemma on the characterization of the classes of the matrix transformations between some classical sequence spaces.

Lemma 2.1. The following statements hold: 
(a) $A=\left(a_{n k}\right) \in\left(c_{0}, c_{0}\right)$ if and only if (2.2) and (2.3) hold.

(b) $A=\left(a_{n k}\right) \in\left(c_{0}, c\right)$ if and only if (2.2) and (2.4) hold.

(c) $A=\left(a_{n k}\right) \in\left(c, c_{0}\right)$ if and only if (2.2), (2.3) and (2.5) hold.

(d) $A=\left(a_{n k}\right) \in(c, c)$ if and only if (2.2), (2.4) and (2.6) hold.

(e) $A=\left(a_{n k}\right) \in\left(c_{0}, \ell_{\infty}\right)=\left(c, \ell_{\infty}\right)$ if and only if the condition (2.2) holds.

(f) $A=\left(a_{n k}\right) \in\left(c_{0}, \ell_{1}\right)=\left(c, \ell_{1}\right)$ if and only if the condition (2.7) holds.

(g) $A=\left(a_{n k}\right) \in\left(\ell_{p}, c\right)$ if and only if (2.4) and (2.8) hold, where $0<$ $p<1$.

(h) $A=\left(a_{n k}\right) \in\left(\ell_{p}, \ell_{\infty}\right)$ if and only if the condition (2.8) holds, where $0<p<1$.

\section{The Fibonacci difference spaces of absolutely $p$-Summable, NULL AND CONVERGENT SEQUENCES}

In this section, we define the spaces $\ell_{p}(\widehat{F}), c_{0}(\widehat{F})$ and $c(\widehat{F})$ of Fibonacci absolutely $p$-summable, Fibonacci null and Fibonacci convergent sequences, where $0<p<1$. Also, we present some inclusion theorems and construct the Schauder bases of the spaces $\ell_{p}(\widehat{F}), c_{0}(\widehat{F})$ and $c(\widehat{F})$.

Recently, Kara [21] has defined the sequence space $\ell_{p}(\widehat{F})$ as follows:

$$
\ell_{p}(\widehat{F})=\left\{x \in \omega: \widehat{F} x \in \ell_{p}\right\},(1 \leq p \leq \infty),
$$

where $\widehat{F}=\left(\widehat{f}_{n k}\right)$ is the double band matrix defined by the sequence $\left(f_{n}\right)$ of Fibonacci numbers as follows

$$
\widehat{f}_{n k}=\left\{\begin{array}{cl}
-\frac{f_{n+1}}{f_{n}} \quad, \quad k=n-1, \\
\frac{f_{n}}{f_{n+1}} \quad, \quad k=n, \\
0 \quad, \quad 0 \leq k<n-1 \text { or } k>n
\end{array}\right.
$$

for all $k, n \in \mathbb{N}$. Also, in [22], Kara et al. have characterized some classes of compact operators on the spaces $\ell_{p}(\widehat{F})$ and $\ell_{\infty}(\widehat{F})$, where $1 \leq p<\infty$.

One can derive by a straightforward calculation that the inverse $\widehat{F}^{-1}=$ $\left(g_{n k}\right)$ of the Fibonacci matrix $\widehat{F}$ is given by

$$
g_{n k}=\left\{\begin{array}{cl}
\frac{f_{n+1}^{2}}{f_{k} f_{k+1}} & , \quad 0 \leq k \leq n \\
0 & , \quad k>n
\end{array}\right.
$$

for all $k, n \in \mathbb{N}$.

Now, we introduce the Fibonacci difference sequence spaces $\ell_{p}(\widehat{F}), c_{0}(\widehat{F})$ and $c(\widehat{F})$ as the set of all sequences whose $\widehat{F}$-transforms are in the spaces 
$\ell_{p}, c_{0}$ and $c$, respectively, i.e.,

$$
\begin{aligned}
\ell_{p}(\widehat{F}) & :=\left\{x=\left(x_{n}\right) \in \omega: \sum_{n}\left|\frac{f_{n}}{f_{n+1}} x_{n}-\frac{f_{n+1}}{f_{n}} x_{n-1}\right|^{p}<\infty\right\},(0<p<1), \\
c_{0}(\widehat{F}): & =\left\{x=\left(x_{n}\right) \in \omega: \lim _{n \rightarrow \infty}\left(\frac{f_{n}}{f_{n+1}} x_{n}-\frac{f_{n+1}}{f_{n}} x_{n-1}\right)=0\right\}, \\
c(\widehat{F}) & :=\left\{x=\left(x_{n}\right) \in \omega: \exists l \in \mathbb{R} \ni \lim _{n \rightarrow \infty}\left(\frac{f_{n}}{f_{n+1}} x_{n}-\frac{f_{n+1}}{f_{n}} x_{n-1}\right)=l\right\} .
\end{aligned}
$$

With the notation of $(1.2)$, the spaces $\ell_{p}(\widehat{F}), c_{0}(\widehat{F})$ and $c(\widehat{F})$ can be redefined as follows:

$$
\ell_{p}(\widehat{F})=\left(\ell_{p}\right)_{\widehat{F}}, \quad c_{0}(\widehat{F})=\left(c_{0}\right)_{\widehat{F}} \quad \text { and } \quad c(\widehat{F})=c_{\widehat{F}} .
$$

Here and after, we assume unless stated otherwise that $0<p<1$ and all the terms with negative subscript are equal to zero.

Define the sequence $y=\left(y_{k}\right)$ by the $\widehat{F}$-transform of a sequence $x=\left(x_{k}\right)$, i.e.,

$$
y_{k}=\widehat{F}_{k}(x)=\frac{f_{k}}{f_{k+1}} x_{k}-\frac{f_{k+1}}{f_{k}} x_{k-1}
$$

for all $k \in \mathbb{N}$. Therefore, one can derive by a straightforward calculation that

$$
x_{k}=\sum_{j=0}^{k} \frac{f_{k+1}^{2}}{f_{j} f_{j+1}} y_{j} \text { for all } k \in \mathbb{N} .
$$

Throughout the text, we suppose that the sequences $x=\left(x_{k}\right)$ and $y=\left(y_{k}\right)$ are connected with the relation (3.1).

Theorem 3.1. The following statements hold:

(i) The sets $\ell_{p}(\widehat{F}), c_{0}(\widehat{F})$ and $c(\widehat{F})$ are the linear spaces with the coordinatewise addition and scalar multiplication.

(ii) $\ell_{p}(\widehat{F})$ is a complete $p$-normed space with the $p$-norm $\|x\|_{p}=$ $\sum_{n}\left|\widehat{F}_{n}(x)\right|^{p}$.

(iii) $c_{0}(\widehat{F})$ and $c(\widehat{F})$ are the BK-spaces with the norm $\|x\|_{c_{0}(\widehat{F})}=\|x\|_{c(\widehat{F})}$ $=\|\widehat{F} x\|_{\infty}$.

Proof. This is a routine verification and so we omit the detail.

Remark 3.2. One can easily check that the absolute property does not hold on the spaces $\ell_{p}(\widehat{F}), c_{0}(\widehat{F})$ and $c(\widehat{F})$, that is $\|x\|_{p} \neq\|x \mid\|_{p},\|x\|_{c_{0}(\widehat{F})} \neq$ $\||x|\|_{c_{0}(\widehat{F})}$ and $\|x\|_{c(\widehat{F})} \neq\||x|\|_{c(\widehat{F})}$ for at least one sequence in the spaces 
$\ell_{p}(\widehat{F}), c_{0}(\widehat{F})$ and $c(\widehat{F})$, and this tells us that $\ell_{p}(\widehat{F}), c_{0}(\widehat{F})$ and $c(\widehat{F})$ are the sequence spaces of non-absolute type, where $|x|=\left(\left|x_{k}\right|\right)$.

Let $\lambda$ denotes any of the spaces $\ell_{p}, c_{0}$ and $c$. With the notation of (3.1), since the transformation $T: \lambda(\widehat{F}) \rightarrow \lambda$ defined by $x \mapsto y=T x=\widehat{F} x$ is a norm preserving linear bijection, we have the following:

Corollary 3.3. The Fibonacci difference sequence spaces $\ell_{p}(\widehat{F}), c_{0}(\widehat{F})$ and $c(\widehat{F})$ of non-absolute type are linearly p-norm/norm isomorphic to the spaces $\ell_{p}, c_{0}$ and $c$, respectively, i.e., $\ell_{p}(\widehat{F}) \cong \ell_{p}, c_{0}(\widehat{F}) \cong c_{0}$ and $c(\widehat{F}) \cong c$.

Now, we give some inclusion relations concerned with the spaces $c_{0}(\widehat{F})$ and $c(\widehat{F})$.

Theorem 3.4. The inclusion $c_{0}(\widehat{F}) \subset c(\widehat{F})$ strictly holds.

Proof. It is clear that the inclusion $c_{0}(\widehat{F}) \subset c(\widehat{F})$ holds. Further, to show the strictness of the inclusion, consider the sequence $x=\left(x_{k}\right)=\left(\sum_{j=0}^{k} f_{k+1}^{2} / f_{j}^{2}\right)$. Then, we obtain by (3.1) for all $k \in \mathbb{N}$ that

$$
\widehat{F}_{k}(x)=\frac{f_{k}}{f_{k+1}} \sum_{j=0}^{k} \frac{f_{k+1}^{2}}{f_{j}^{2}}-\frac{f_{k+1}}{f_{k}} \sum_{j=0}^{k-1} \frac{f_{k}^{2}}{f_{j}^{2}}=\frac{f_{k+1}}{f_{k}}
$$

which tends to $\varphi$, as $k \rightarrow \infty$ by (2.1). That is to say that $\widehat{F} x \in c \backslash c_{0}$. Thus, the sequence $x$ is in $c(\widehat{F})$ but is not in $c_{0}(\widehat{F})$. Hence, the inclusion $c_{0}(\widehat{F}) \subset c(\widehat{F})$ is strict.

Theorem 3.5. The space $\ell_{\infty}$ does not include the spaces $c_{0}(\widehat{F})$ and $c(\widehat{F})$.

Proof. Let us consider the sequence $x=\left(x_{k}\right)=\left(f_{k+1}^{2}\right)$. Since $f_{k+1}^{2} \rightarrow \infty$, as $k \rightarrow \infty$ and $\widehat{F} x=e^{(0)}=(1,0,0, \ldots)$; the sequence $x$ is in the space $c_{0}(\widehat{F})$ but is not in the space $\ell_{\infty}$. This shows that the space $\ell_{\infty}$ does not include both the space $c_{0}(\widehat{F})$ and the space $c(\widehat{F})$, as desired.

Theorem 3.6. The inclusions $c_{0} \subset c_{0}(\widehat{F})$ and $c \subset c(\widehat{F})$ strictly hold.

Proof. Let $\lambda=c_{0}$ or $c$. Since the matrix $\widehat{F}=\left(\widehat{f}_{n k}\right)$ satisfies the conditions

$$
\begin{aligned}
& \sup _{n \in \mathbb{N}} \sum_{k}\left|\widehat{f}_{n k}\right|=\sup _{n \in \mathbb{N}}\left(\frac{f_{n}}{f_{n+1}}+\frac{f_{n+1}}{f_{n}}\right)=2+\frac{1}{2}=\frac{5}{2}, \\
& \lim _{n \rightarrow \infty} \widehat{f}_{n k}=0, \\
& \lim _{n \rightarrow \infty} \sum_{k} \widehat{f}_{n k}=\lim _{n \rightarrow \infty}\left(\frac{f_{n}}{f_{n+1}}-\frac{f_{n+1}}{f_{n}}\right)=\frac{1}{\varphi}-\varphi
\end{aligned}
$$


we conclude by Parts (a) and (d) of Lemma 2.1 that $\widehat{F} \in(\lambda, \lambda)$. This leads to the fact that $\widehat{F} x \in \lambda$ for any $x \in \lambda$. Thus, $x \in \lambda(\widehat{F})$. This shows that $\lambda \subset \lambda(\widehat{F})$.

Now, let $x=\left(x_{k}\right)=\left(f_{k+1}^{2}\right)$. Then, it is clear that $x \in \lambda(\widehat{F}) \backslash \lambda$. This says that the inclusion $\lambda \subset \lambda(\widehat{F})$ is strict.

Theorem 3.7. The spaces $c_{0}(\widehat{F})$ and $c(\widehat{F})$ are not solid.

Proof. Consider the sequences $u=\left(u_{k}\right)$ and $v=\left(v_{k}\right)$ defined by $u_{k}=f_{k+1}^{2}$ and $v_{k}=(-1)^{k+1}$ for all $k \in \mathbb{N}$. Then, it is clear that $u \in c_{0}(\widehat{F})$ and $v \in \ell_{\infty}$. Nevertheless $u v=\left\{(-1)^{k+1} f_{k+1}^{2}\right\}$ is not in the space $c_{0}(\widehat{F})$, since

$$
\widehat{F}_{k}(u v)=\frac{f_{k}}{f_{k+1}}(-1)^{k+1} f_{k+1}^{2}-\frac{f_{k+1}}{f_{k}}(-1)^{k} f_{k}^{2}=2(-1)^{k+1} f_{k} f_{k+1}
$$

for all $k \in \mathbb{N}$. This shows that the multiplication $\ell_{\infty} c_{0}(\widehat{F})$ of the spaces $\ell_{\infty}$ and $c_{0}(\widehat{F})$ is not a subset of $c_{0}(\widehat{F})$. Hence, the space $c_{0}(\widehat{F})$ is not solid.

It is clear here that if the space $c_{0}(\widehat{F})$ is replaced by the space $c(\widehat{F})$, then we obtain the fact that $c(\widehat{F})$ is not solid. This completes the proof.

It is known from Theorem 2.3 of Jarrah and Malkowsky [19] that if $T$ is a triangle then the domain $\lambda_{T}$ of $T$ in a normed sequence space $\lambda$ has a basis if and only if $\lambda$ has a basis. As a direct consequence of this fact, we have:

Corollary 3.8. Define the sequences $c^{(-1)}=\left\{c_{k}^{(-1)}\right\}_{k \in \mathbb{N}}$ and $c^{(n)}=\left\{c_{k}^{(n)}\right\}_{k \in \mathbb{N}}$ for every fixed $n \in \mathbb{N}$ by

$$
c_{k}^{(-1)}=\sum_{j=0}^{k} \frac{f_{k+1}^{2}}{f_{j} f_{j+1}} \quad \text { and } \quad c_{k}^{(n)}:=\left\{\begin{array}{cl}
0 & , \quad 0 \leq k \leq n-1, \\
\frac{f_{k+1}^{2}}{f_{n} f_{n+1}} \quad, \quad k \geq n .
\end{array}\right.
$$

Then, the following statements hold:

(a) The sequence $\left\{c^{(n)}\right\}_{n=0}^{\infty}$ is a basis for the spaces $\ell_{p}(\widehat{F})$ and $c_{0}(\widehat{F})$, and every sequence $x \in c_{0}(\widehat{F})$ or in the space $\ell_{p}(\widehat{F})$, has a unique representation of the form $x=\sum_{n} \widehat{F}_{n}(x) c^{(n)}$.

(b) The sequence $\left\{c^{(n)}\right\}_{n=-1}^{\infty}$ is a basis for the space $c(\widehat{F})$ and every sequence $z=\left(z_{n}\right) \in c(\widehat{F})$ has a unique representation of the form $z=l c^{(-1)}+\sum_{n}\left[\widehat{F}_{n}(z)-l\right] c^{(n)}$, where $l=\lim _{n \rightarrow \infty} \widehat{F}_{n}(z)$. 
4. The Alpha, Beta AND Gamma DuAls of The SPACES $\ell_{p}(\widehat{F}), c_{0}(\widehat{F})$ AND $c(\widehat{F})$, AND SOME MATRIX TRANSFORMATIONS

In this section, we determine the alpha, beta and gamma duals of the spaces $\ell_{p}(\widehat{F}), c_{0}(\widehat{F})$ and $c(\widehat{F})$, and characterize the classes of infinite matrices from the spaces $\ell_{p}(\widehat{F})$ to the spaces $\ell_{\infty}, c$ and $c_{0}$.

Theorem 4.1. The alpha dual of the spaces $c_{0}(\widehat{F})$ and $c(\widehat{F})$ is the set

$$
d_{1}:=\left\{a=\left(a_{k}\right) \in \omega: \sup _{K \in \mathcal{F}} \sum_{n}\left|\sum_{k \in K} b_{n k}\right|<\infty\right\},
$$

where the matrix $B=\left(b_{n k}\right)$ is defined by

$$
b_{n k}:=\left\{\begin{array}{cl}
\frac{f_{n+1}^{2}}{f_{k} f_{k+1}} a_{n} & , \quad 0 \leq k \leq n, \\
0 & , \quad k>n
\end{array}\right.
$$

for all $k, n \in \mathbb{N}$.

Proof. Let $a=\left(a_{n}\right) \in \omega$. Consider the equality

$$
a_{n} x_{n}=\sum_{k=0}^{n} \frac{f_{n+1}^{2}}{f_{k} f_{k+1}} a_{n} y_{k}=\sum_{k=0}^{n} b_{n k} y_{k}=B_{n}(y) .
$$

By (4.1), we obtain that $a x=\left(a_{n} x_{n}\right) \in \ell_{1}$ whenever $x=\left(x_{k}\right) \in c_{0}(\widehat{F})$ or $c(\widehat{F})$ if and only if $B y \in \ell_{1}$ whenever $y=\left(y_{k}\right) \in c_{0}$ or $c$. That is, the sequence $a=\left(a_{n}\right)$ is in the alpha dual of the space $c_{0}(\widehat{F})$ or $c(\widehat{F})$ if and only if $B \in\left(c_{0}, \ell_{1}\right)=\left(c, \ell_{1}\right)$. By taking $B$ instead of $A$ in part (f) of Lemma 2.1, we obtain that $a \in\left[c_{0}(\widehat{F})\right]^{\alpha}=[c(\widehat{F})]^{\alpha}$ if and only if

$$
\sup _{K \in \mathcal{F}} \sum_{n}\left|\sum_{k \in K} b_{n k}\right|<\infty
$$

which means that $\left[c_{0}(\widehat{F})\right]^{\alpha}=[c(\widehat{F})]^{\alpha}=d_{1}$.

Theorem 4.2. Define the sets $d_{2}, d_{3}, d_{4}$ and $d_{5}$, as follows:

$$
\begin{aligned}
& d_{2}:=\left\{a=\left(a_{k}\right) \in \omega: \sup _{n \in \mathbb{N}} \sum_{k=0}^{n}\left|\sum_{j=k}^{n} \frac{f_{j+1}^{2}}{f_{k} f_{k+1}} a_{j}\right|<\infty\right\}, \\
& d_{3}:=\left\{a=\left(a_{k}\right) \in \omega: \lim _{n \rightarrow \infty} \sum_{j=k}^{n} \frac{f_{j+1}^{2}}{f_{k} f_{k+1}} a_{j} \text { exists for each } k \in \mathbb{N}\right\}, \\
& d_{4}:=\left\{a=\left(a_{k}\right) \in \omega: \lim _{n \rightarrow \infty} \sum_{k=0}^{n} \sum_{j=k}^{n} \frac{f_{j+1}^{2}}{f_{k} f_{k+1}} a_{j} \text { exists }\right\},
\end{aligned}
$$




$$
d_{5}:=\left\{a=\left(a_{k}\right) \in \omega: \sup _{k, n \in \mathbb{N}}\left|\sum_{j=k}^{n} \frac{f_{j+1}^{2}}{f_{k} f_{k+1}} a_{j}\right|<\infty\right\} .
$$

Then, the following statements hold:

(a) $\left[c_{0}(\widehat{F})\right]^{\alpha}=[c(\widehat{F})]^{\alpha}=d_{1}$.

(b) $\left[c_{0}(\widehat{F})\right]^{\beta}=d_{2} \cap d_{3}$.

(c) $[c(\widehat{F})]^{\beta}=d_{2} \cap d_{3} \cap d_{4}$.

(d) $\left[\ell_{p}(\widehat{F})\right]^{\beta}=d_{3} \cap d_{5}$.

(e) $\left[c_{0}(\widehat{F})\right]^{\gamma}=[c(\widehat{F})]^{\gamma}=d_{2}$.

(f) $\left[\ell_{p}(\widehat{F})\right]^{\gamma}=d_{5}$.

Proof. Consider the equality

$$
\sum_{k=0}^{n} a_{k} x_{k}=\sum_{k=0}^{n} a_{k}\left(\sum_{i=0}^{k} \frac{f_{k+1}^{2}}{f_{i} f_{i+1}} y_{i}\right)=\sum_{k=0}^{n}\left(\sum_{i=k}^{n} \frac{f_{i+1}^{2}}{f_{k} f_{k+1}} a_{i}\right) y_{k}=C_{n}(y),
$$

where $C=\left(c_{n k}\right)$ defined by

$$
c_{n k}:=\left\{\begin{array}{cl}
\sum_{i=k}^{n} \frac{f_{i+1}^{2}}{f_{k} f_{k+1}} a_{i} & , \quad 0 \leq k \leq n \\
0 & , \quad k>n
\end{array}\right.
$$

for all $k, n \in \mathbb{N}$. Then, we observe by (4.2) that $a x=\left(a_{n} x_{n}\right) \in c s$ whenever $x \in c_{0}(\widehat{F})$ if and only if $C y \in c$ whenever $y=\left(y_{k}\right) \in c_{0}$ which means that $a \in\left[c_{0}(\widehat{F})\right]^{\beta}$ if and only if $C \in\left(c_{0}, c\right)$. By using Part (b) of Lemma 2.1, we derive that

$$
\begin{aligned}
& \sup _{n \in \mathbb{N}} \sum_{k}\left|\sum_{i=k}^{n} \frac{f_{i+1}^{2}}{f_{k} f_{k+1}} a_{i}\right|<\infty, \\
& \lim _{n \rightarrow \infty} \sum_{i=k}^{n} \frac{f_{i+1}^{2}}{f_{k} f_{k+1}} a_{i} \text { exists for each } k \in \mathbb{N} .
\end{aligned}
$$

Hence, we conclude that $\left[c_{0}(\widehat{F})\right]^{\beta}=d_{2} \cap d_{3}$.

In a similar way, one can easily show the facts about the beta duals of the spaces $c(\widehat{F}), \ell_{p}(\widehat{F})$ and gamma duals of the spaces $c_{0}(\widehat{F}), c(\widehat{F}), \ell_{p}(\widehat{F})$.

Now, we give the theorems characterizing the classes $\left(\ell_{p}(\widehat{F}): \ell_{\infty}\right),\left(\ell_{p}(\widehat{F})\right.$ : c) and $\left(\ell_{p}(\widehat{F}): c_{0}\right)$ of infinite matrices.

Theorem 4.3. $A=\left(a_{n k}\right) \in\left(\ell_{p}(\widehat{F}): \ell_{\infty}\right)$ if and only if

$$
\sup _{k, n \in \mathbb{N}}\left|\sum_{j=k}^{\infty} \frac{f_{j+1}^{2}}{f_{k} f_{k+1}} a_{n j}\right|<\infty \text {. }
$$


Proof. $A=\left(a_{n k}\right) \in\left(\ell_{p}(\widehat{F}): \ell_{\infty}\right)$ and $x=\left(x_{k}\right) \in \ell_{p}(\widehat{F})$. Then, $A x$ exists and belongs to the space $\ell_{\infty}$. Therefore, one can conclude for $x^{(k)}=\left\{x_{j}^{(k)}\right\} \in$ $\ell_{p}(\widehat{F})$ defined by

$$
x_{j}^{(k)}:=\left\{\begin{array}{cl}
f_{j+1}^{2} / f_{k} f_{k+1} & , \quad j \geq k \\
0 & , \quad 0 \leq j \leq k-1
\end{array}\right.
$$

for all $j, k \in \mathbb{N}$ that $A x^{(k)}=\left(\sum_{j=k}^{\infty} f_{j+1}^{2} a_{n j} / f_{k} f_{k+1}\right) \in \ell_{\infty}$ for each $k \in \mathbb{N}$. Hence, the condition (4.3) is necessary.

Conversely, suppose that (4.3) holds and take any $x=\left(x_{k}\right) \in \ell_{p}(\widehat{F})$. Then, $A_{n} \in\left[\ell_{p}(\widehat{F})\right]^{\beta}$ for each $n \in \mathbb{N}$ which leads to the existence of $A x$. Let $n \in \mathbb{N}$ be fixed. Consider the following relation derived from the $m^{\text {th }}$ partial sum of the series $\sum_{k} a_{n k} x_{k}$ with (3.2):

$$
\sum_{k=0}^{m} a_{n k} x_{k}=\sum_{k=0}^{m} \sum_{j=k}^{m} \frac{f_{j+1}^{2}}{f_{k} f_{k+1}} a_{n j} y_{k}
$$

for all $m, n \in \mathbb{N}$. Then, by letting $m \rightarrow \infty$ in (4.5) we have

$$
A_{n}(x)=\sum_{k} a_{n k} x_{k}=\sum_{k} e_{n k} y_{k}=E_{n}(y)
$$

for all $n \in \mathbb{N}$, where the matrix $E=\left(e_{n k}\right)$ is defined by

$$
e_{n k}=\sum_{j=k}^{\infty} \frac{f_{j+1}^{2}}{f_{k} f_{k+1}} a_{n j}
$$

for all $k, n \in \mathbb{N}$. Therefore, since

$$
\begin{aligned}
\left|\sum_{k} a_{n k} x_{k}\right|^{p} & =\left|\sum_{k} \sum_{j=k}^{\infty} \frac{f_{j+1}^{2}}{f_{k} f_{k+1}} a_{n j} y_{k}\right|^{p} \\
& \leq\left(\sum_{k}\left|\sum_{j=k}^{\infty} \frac{f_{j+1}^{2}}{f_{k} f_{k+1}} a_{n j}\right|\left|y_{k}\right|\right)^{p} \\
& \leq\left(\sup _{k \in \mathbb{N}}\left|\sum_{j=k}^{\infty} \frac{f_{j+1}^{2}}{f_{k} f_{k+1}} a_{n j}\right|\right)^{p}\left(\sum_{k}\left|y_{k}\right|\right)^{p} \\
& \leq\left(\sup _{k \in \mathbb{N}}\left|\sum_{j=k}^{\infty} \frac{f_{j+1}^{2}}{f_{k} f_{k+1}} a_{n j}\right|\right)^{p} \sum_{k}\left|y_{k}\right|^{p}
\end{aligned}
$$


we obtain by taking supremum over $n \in \mathbb{N}$ that

$$
\|A x\|_{\infty}=\sup _{n \in \mathbb{N}}\left|\sum_{k} a_{n k} x_{k}\right| \leq\left(\|y\|_{p}\right)^{1 / p} \sup _{k, n \in \mathbb{N}}\left|\sum_{j=k}^{\infty} \frac{f_{j+1}^{2}}{f_{k} f_{k+1}} a_{n j}\right|<\infty .
$$

That is, $A x \in \ell_{\infty}$, as desired.

This completes the proof.

Theorem 4.4. $A=\left(a_{n k}\right) \in\left(\ell_{p}(\widehat{F}): c\right)$ if and only if (4.3) holds, and

$$
\exists \alpha_{k} \in \mathbb{R} \text { such that } \lim _{n \rightarrow \infty} \sum_{j=k}^{\infty} \frac{f_{j+1}^{2}}{f_{k} f_{k+1}} a_{n j}=\alpha_{k} \text { for each } k \in \mathbb{N} .
$$

Proof. Let $A=\left(a_{n k}\right) \in\left(\ell_{p}(\widehat{F}): c\right)$. Then, $A x$ exists and is in the space $c$ for all $x=\left(x_{k}\right) \in \ell_{p}(\widehat{F})$. Since the inclusion $c \subset \ell_{\infty}$ holds, the necessity of the condition (4.3) follows from Theorem 4.3. The necessity of the condition (4.8) is immediate by taking the sequence $x^{(k)}=\left\{x_{j}^{(k)}\right\} \in \ell_{p}(\widehat{F})$ defined by (4.4).

Conversely, suppose that the conditions (4.3) and (4.8) hold, and take any $x=\left(x_{k}\right) \in \ell_{p}(\widehat{F})$. Then, since $A_{n} \in\left\{\ell_{p}(\widehat{F})\right\}^{\beta}$ for each $n \in \mathbb{N}, A x$ exists. Then, by taking into account the relation (4.6) one can see that the conditions (4.3) and (4.8) correspond to (2.8) and (2.4) with $e_{n k}$ instead of $a_{n k}$, respectively; where $e_{n k}$ is given by (4.7). Hence, $E y \in c$ which gives by (4.6) that $A \in\left(\ell_{p}(\widehat{F}): c\right)$.

If we replace the space $c_{0}$ with the space $c$, then Theorem 4.4 yields the following:

Corollary 4.5. $A=\left(a_{n k}\right) \in\left(\ell_{p}(\widehat{F}): c_{0}\right)$ if and only if (4.3) holds, and (4.8) also holds with $\alpha_{k}=0$ for all $k \in \mathbb{N}$.

Theorem 4.6. Suppose that the elements of the infinite matrices $A=\left(a_{n k}\right)$ and $H=\left(h_{n k}\right)$ are connected by the relation

$$
h_{n k}=-\frac{f_{n+1}}{f_{n}} a_{n-1, k}+\frac{f_{n}}{f_{n+1}} a_{n k}
$$

for all $k, n \in \mathbb{N}$ and $\mu$ be any given sequence space. Then, $A \in(\mu, \lambda(\widehat{F}))$ if and only if $H \in(\mu, \lambda)$; where $\lambda$ denotes any of the classical sequence spaces $\ell_{p}, c_{0}$ or $c$.

Proof. Let $z=\left(z_{k}\right) \in \mu$. Then, by taking into account the relation (4.9) one can easily derive the following equality

$$
\sum_{k=0}^{m} h_{n k} z_{k}=\sum_{k=0}^{m}\left(-\frac{f_{n+1}}{f_{n}} a_{n-1, k}+\frac{f_{n}}{f_{n+1}} a_{n k}\right) z_{k} \text { for all } m, n \in \mathbb{N}
$$


which yields as $m \rightarrow \infty$ that $H_{n}(z)=(\widehat{F} A)_{n}(z)$. Therefore, we conclude that $A z \in \lambda(\widehat{F})$ whenever $z \in \mu$ if and only if $H z \in \lambda$ whenever $z \in \mu$. This step completes the proof.

It is trivial that combining Theorems $4.3,4.4$ and Corollary 4.5 with Theorem 4.6, one can derive the following results:

Corollary 4.7. Let $A=\left(a_{n k}\right)$ be an infinite matrix and $a(n, k)=\sum_{j=0}^{n} a_{j k}$ for all $k, n \in \mathbb{N}$. Then, the following statements hold:

(a) $A=\left(a_{n k}\right) \in\left(\ell_{p}(\widehat{F}), b s\right)$ if and only if $(4.3)$ holds with a $(n, k)$ instead of $a_{n k}$.

(b) $A=\left(a_{n k}\right) \in\left(\ell_{p}(\widehat{F}), c s\right)$ if and only if (4.3)) and (4.8) hold with $a(n, k)$ instead of $a_{n k}$.

(c) $A=\left(a_{n k}\right) \in\left(\ell_{p}(\widehat{F}), c s_{0}\right)$ if and only if (4.3) and (4.8) hold with $a(n, k)$ instead of $a_{n k}$ with $\alpha_{k}=0$ for all $k \in \mathbb{N}$, where $c s_{0}$ denotes the space of series converging to zero.

(d) $A=\left(a_{n k}\right) \in\left(\ell_{p}(\widehat{F}), \ell_{\infty}(\widehat{F})\right)$ if and only if (4.3) holds with $h_{n k}$ instead of $a_{n k}$.

(e) $A=\left(a_{n k}\right) \in\left(\ell_{p}(\widehat{F}), c(\widehat{F})\right)$ if and only if (4.3) and (4.8) hold with $h_{n k}$ instead of $a_{n k}$.

(f) $A=\left(a_{n k}\right) \in\left(\ell_{p}(\widehat{F}), c_{0}(\widehat{F})\right)$ if and only if (4.3) and (4.8) hold with $h_{n k}$ instead of $a_{n k}$ with $\alpha_{k}=0$ for all $k \in \mathbb{N}$.

\section{Conclusion}

To review the relevant literature about the domain of the infinite matrix $A$ in the sequence spaces $\ell_{p}, c_{0}$ and $c$, the table on the next page may be useful.

The present paper is devoted to the sequence spaces $\ell_{p}(\widehat{F}), c_{0}(\widehat{F})$ and $c(\widehat{F})$ obtained as the domain of the double band matrix $\widehat{F}$ in the classical spaces $\ell_{p}, c_{0}$ and $c$, respectively. Of course, the $\alpha-, \beta$ - and $\gamma$-duals of the spaces $c_{0}(\widehat{F}), c(\widehat{F})$ and $\ell_{p}(\widehat{F})$ with $0<p<1$ can be given, indirectly, in the light of Theorem 3.1 of Altay and Başar [3]. However, we prefer to do this by following the similar approach used in the proof of Theorems 4.1-4.3 of Başar and Altay [8].

We should state that although the double sequential band matrix $B(\widetilde{r}, \widetilde{s})$ can be reduced to the matrix $\widehat{F}$ in the case $\widetilde{r}=\left(r_{n}\right)$ and $\widetilde{s}=\left(s_{n}\right)$ with $r_{n}=$ $f_{n} / f_{n+1}$ and $s_{n-1}=-f_{n+1} / f_{n}$ for all $n \in \mathbb{N}$, the main results concerning the spaces $c_{0}(\widehat{F})$ and $c(\widehat{F})$ are obtained independently from Candan [13]. It is worth mentioning here that in spite of the domain of the matrix $\widehat{F}$ in the space $\ell_{p}$ of absolutely $p$-summable sequences has been recently studied by 
Kara in [21] for $1 \leq p<\infty$, our results related to the space $\ell_{p}(\widehat{F})$ are new and are also complementary of $[21]$.

\begin{tabular}{|c|c|c|c|}
\hline$A$ & $\lambda$ & $\lambda_{A}$ & refer to: \\
\hline$\Delta$ & $c_{0}$ and $c$ & $c_{0}(\Delta)$ and $c(\Delta)$ & {$[23]$} \\
$\Delta$ & $\ell_{p},(0<p \leq 1)$ & $b v_{p}$ & {$[4]$} \\
$B(r, s, t)$ & $c_{0}$ and $c$ & $B\left(c_{0}\right)$ and $B(c)$ & {$[33]$} \\
$C_{1}$ & $c_{0}$ and $c$ & $\widetilde{c}_{0}$ and $\widetilde{c}$ & {$[35]$} \\
$A^{r}$ & $c_{0}$ and $c$ & $a_{0}^{r}$ and $a_{c}^{r}$ & {$[6]$} \\
$\Delta^{2}$ & $c_{0}$ and $c$ & $c_{0}\left(\Delta^{2}\right)$ and $c\left(\Delta^{2}\right)$ & {$[15]$} \\
$u \Delta^{2}$ & $c_{0}$ and $c$ & $c_{0}\left(u ; \Delta^{2}\right)$ and $c\left(u ; \Delta^{2}\right)$ & {$[30]$} \\
$\Delta^{m}$ & $c_{0}$ and $c$ & $c_{0}\left(\Delta^{m}\right)$ and $c\left(\Delta^{m}\right)$ & {$[16,11]$} \\
$R^{q}$ & $c_{0}$ and $c$ & $(\bar{N}, q)_{0}$ and $(\bar{N}, q)$ & {$[26]$} \\
$\Delta^{(m)}$ & $c_{0}$ and $c$ & $c_{0}\left(\Delta^{(m)}\right)$ and $c\left(\Delta^{(m)}\right)$ & {$[27]$} \\
$G(u, v)$ & $c_{0}$ and $c$ & $c_{0}(u, v)$ and $c(u, v)$ & {$[5]$} \\
$\Lambda$ & $c_{0}$ and $c$ & $c_{0}^{\lambda}$ and $c^{\lambda}$ & {$[31]$} \\
$B(r, s)$ & $c_{0}$ and $c$ & $\widehat{c}_{0}$ and $\widehat{c}$ & {$[24]$} \\
$E^{r}$ & $c_{0}$ and $c$ & $e_{0}^{r}$ and $e_{c}^{r}$ & {$[1]$} \\
$A_{\lambda}$ & $c_{0}$ and $c$ & $A_{\lambda}\left(c_{0}\right)$ and $A_{\lambda}(c)$ & {$[10]$} \\
$B(\widetilde{r}, \widetilde{s})$ & $c_{0}$ and $c$ & $\widetilde{c}_{0}$ and $\widetilde{c}$ & {$[13]$} \\
$N^{t}$ & $c_{0}$ and $c$ & $c_{0}\left(N^{t}\right)$ and $c\left(N^{t}\right)$ & {$[36]$} \\
\hline
\end{tabular}

Table 1: The domains of some triangle matrices in the spaces $\ell_{p}, c_{0}$ and $c$

Acknowledgements. The second author would like to thank Prof. Eberhard Malkowsky for his valuable suggestions in proving the sufficiency part of Theorem 4.3 on the earlier version of the manuscript. The authors would like to thank Dr. Medine Yeşilkayagil for her careful reading and pointing out some mistakes and misprints in the earlier version of this paper which improved the presentation and readability. Finally, the authors are grateful to the anonymous referee for his/her helpful comments and valuable suggestions which helped them to improve this paper.

\section{REFERENCES}

[1] B. Altay and F. Başar, Some Euler sequence spaces of non-absolute type, Ukrainian Math. J., 57 (1) (2005), 1-17.

[2] B. Altay and F. Başar, Some paranormed Riezs sequence spaces of non-absolute type, Southeast Asian Bull. Math., 30 (5) (2006), 591-608.

[3] B. Altay and F. Başar, Certain topological properties and duals of the matrix domain of a triangle matrix in a sequence space, J. Math. Anal. Appl., 336 (1) (2007), 632-645

[4] B. Altay and F. Başar, The fine spectrum and the matrix domain of the difference operator $\Delta$ on the sequence space $\ell_{p},(0<p<1)$, Commun. Math. Anal., 2 (2) (2007), 1-11. 
[5] B. Altay and F. Başar, Some paranormed sequence spaces of non-absolute type derived by weighted mean, J. Math. Anal. Appl., 319 (2) (2006), 494-508.

[6] C. Aydın and F. Başar, On the new sequence spaces which include the spaces $c_{0}$ and c, Hokkaido Math. J., 33 (2) (2004), 383-398.

[7] F. Başar, Summability Theory and Its Applications, Bentham Science Publishers, e-books, Monographs, İstanbul, 2012.

[8] F. Başar and B. Altay, On the space of sequences of p-bounded variation and related matrix mappings, Ukrainian Math. J., 55 (2003), 136-147.

[9] Ç.A. Bektaş, M. Et and R. Çolak, Generalized difference sequence spaces and their dual spaces, J. Math. Anal. Appl., 292 (2) (2004), 423-432.

[10] N. L. Braha and F. Başar, On the domain of the triangle $A(\lambda)$ on the spaces of null, convergent and bounded sequences, Abstr. Appl. Anal., 2013, Article ID 476363, 9 pages, 2013, doi:10.1155/2013/476363.

[11] R. Çolak and M. Et, On some generalized difference sequence spaces and related matrix transformations, Hokkaido Math. J., 26 (3) (1997), 483-492.

[12] R. Çolak, M. Et and E. Malkowsky, Some Topics of Sequence Spaces, in: Lecture Notes in Mathematics, Firat Univ. Press (2004), 1-63 ISBN: 975-394-0386-6.

[13] M. Candan, Domain of the double sequential band matrix in the classical sequence spaces, J. Inequal. Appl., 2012:281, 12 pp.

[14] B. Choudhary and S. K. Mishra, A note on Köthe-Toeplitz duals of certain sequence spaces and their matrix transformations, Internat. J. Math. Math. Sci., 18 (4) (1995), $681-688$.

[15] M. Et, On some difference sequence spaces, Turkish J. Math., 17 (1993), 18-24.

[16] M. Et and R. Çolak, On some generalized difference sequence spaces, Soochow J. Math., 21 (4) (1995), 377-386.

[17] M. Et and A. Esi, On Köthe-Toeplitz duals of generalized difference sequence spaces, Bull. Malaysian Math. Sc. Soc., 23 (2000), 25-32.

[18] A. K. Gaur and M. Mursaleen, Difference sequence spaces, Int. J. Math. Math. Sci., 21 (4) (1998), 701-706.

[19] A. M. Jarrah and E. Malkowsky, BK spaces, bases and linear operators, Rendiconti Circ. Mat. Palermo II, 52 (1990), 177-191.

[20] P. K. Kamthan and M. Gupta, Sequence Spaces and Series, Marcel Dekker Inc., New York and Basel, 1981.

[21] E. E. Kara, Some topological and geometrical properties of new Banach sequence spaces, J. Inequal. Appl., 2013:38 15 pages, 2013. doi:10.1186/1029-242X-2013-38.

[22] E. E. Kara, M. Başarır and M. Mursaleen, Compact operators on the Fibonacci difference sequence spaces $\ell_{p}(\widehat{F})$ and $\ell_{\infty}(\widehat{F})$, 1st International Eurasian Conference on Mathematical Sciences and Applications, Prishtine-Kosovo, September 3-7, 2012.

[23] H. Kızmaz, On certain sequence spaces, Canad. Math. Bull., 24 (2) (1981), 169-176.

[24] M. Kirişçi and F. Başar, Some new sequence spaces derived by the domain of generalized difference matrix, Comput. Math. Appl., 60 (5) (2010), 1299-1309.

[25] T. Koshy, Fibonacci and Lucas Numbers with Applications, Wiley, 2001.

[26] E. Malkowsky, Recent results in the theory of matrix transformations in the sequence spaces, Mat. Vesnik, 49 (1997), 187-196.

[27] E. Malkowsky and S.D. Parashar, Matrix transformations in space of bounded and convergent difference sequences of order m, Analysis, 17 (1997), 87-97.

[28] E. Malkowsky and E. Savaş, Matrix transformations between sequence spaces of generalized weighted mean, Appl. Math. Comput., 147 (2004), 333-345. 
[29] S. K. Mishra, Matrix maps involving certain sequence spaces, Indian J. Pure Appl. Math., 24 (2) (1993), 125-132.

[30] M. Mursaleen, Generalized spaces of difference sequences, J. Math. Anal. Appl., 203 (3) (1996), 738-745.

[31] M. Mursaleen and A. K. Noman, On the spaces of $\lambda$-convergent and bounded sequences, Thai J. Math., 8 (2) (2010), 311-329.

[32] M. A. Sarı̈öl, On difference sequence spaces, J. Karadeniz Tech. Univ. Fac. Arts Sci. Ser. Math. -Phys., 10 (1987), 63-71.

[33] A. Sönmez, Some new sequence spaces derived by the domain of the triple band matrix, Comput. Math. Appl., 62 (2) (2011), 641-650.

[34] A. Sönmez, Almost convergence and triple band matrix, Math. Comput. Model., 57 (9-10) (2013), 2393-2402.

[35] M. Şengönül and F. Başar, Some new Cesàro sequence spaces of non-absolute type which include the spaces $c_{0}$ and c, Soochow J. Math., 31 (1) (2005), 107-119.

[36] O. Tuğ and F. Başar, On the domain of Nörlund mean in the spaces of null and convergent sequences, TWMS J. Pure Appl. Math., 7 (1) (2016), 76-87.

(Received: June 24, 2015)

(Revised: January 22, 2016)

\author{
Metin Başarır \\ Sakarya Üniversitesi \\ Fen-Edebiyat Fakültesi \\ Matematik Bölümü \\ Esentepe Kampüsü, Sakarya-54187, \\ Türkiye \\ basarir@sakarya.edu.tr \\ Feyzi Başar \\ Kısıklı Mah. Alim Sok. \\ Alim Apt. No. 7/6 \\ 34692 Üsküdar İstanbul \\ Türkiye \\ feyzibasar@gmail.com \\ Emrah Evren Kara \\ Düzce Üniversitesi, \\ Fen-Edebiyat Fakültesi \\ Matematik Bölümü \\ Konuralp Yerleşkesi, Düzce-81620 \\ Türkiye \\ eevrenkara@hotmail.com
}

